\title{
Quasi-Fixed-Frequency Hysteresis Current Tracking Control Strategy for Modular Multilevel Converters
}

\author{
Jun $\mathrm{Mei}^{\dagger}$, Yu Ji ${ }^{*}$, Xiaozhou Du* ${ }^{*}$ Tian $\mathrm{Ma}^{*}$, Can Huang ${ }^{* *}$, and Qinran $\mathrm{Hu}^{* *}$ \\ $\dot{\dagger}^{*}$ Dept. of Electrical Engineering, Southeast University, Jiangsu, China \\ ${ }^{* *}$ Dept. of Electrical Engineering \& Computer Science, the University of Tennessee, Knoxville, USA
}

\begin{abstract}
This study proposes a quasi-fixed-frequency hysteresis current tracking control strategy for modular multilevel converters (MMCs) on the basis of voltage partition principle. First, by monitoring the grid voltage and the deviation between the output and reference currents, the output voltage is determined, thus prompting the output current to quickly and efficiently track the given current. Second, the voltages of the upper/lower capacitor of the arm and the voltages between the upper and lower arms are balanced by combining these arms with virtual loop mapping and arm voltage balance control, respectively. In particular, the proposed method is designed for any level and number of sub-modules. The validity of the proposed method is verified by simulations and experimental results of a five-level MMC prototype.
\end{abstract}

Key words: Dynamic voltage balance, Modular multilevel converter (MMC), Quasi-fixed-frequency hysteresis current tracking control, Virtual loop mapping

\section{INTRODUCTION}

Modular multilevel converter (MMC) is an innovative multilevel technology that was first used in a high voltage direct current (HVDC) transmission project in 2010 [1]. Compared with conventional multilevel topologies, MMC offers several advantages, including low switching frequency, low harmonic, and favorable redundancy. Also, as a new topology based on double-star chopper cell, MMC presents numerous desirable features, such as adaptability to high -voltage and high-power applications, excellent four-quadrant performance, and modular structure and redundant design [2], [3].

To fully utilize the favorable features of MMC, considerable effort has been exerted to extend the applications of MMC from HVDC [4]-[6] to various fields, including high-power motor drives [7], medium-voltage STATCOM [8], [9], and renewable energy generation [10]. However, only a few reports have been published on MMC for active power filters (APFs). As a type of static power

Manuscript received May 12, 2014; accepted Oct. 4, 2014

Recommended for publication by Associate Editor Dong-Wook Yoo.

†Corresponding Author: meijun2000@gmail.com

Tel: +86-025-83794163-606, Southeast University

*Dept. of Electrical Eng., Southeast University, China

** Dept. of Electrical Eng. \& Computer Sci., the Univ. of Tennessee, USA converter, APF is normally controlled to compensate for the current harmonics drained from non-linear loads, reactive power, and imbalances of load terminals [11]. The dynamic performance of APF essential and largely depends on two factors, namely, real-time harmonic detection and rapid output current response. The former has been efficiently solved by instantaneous power theory, whereas the latter still requires sophisticated modulation methods.

This specific challenge has been addressed in the related literature with the implementation of several advanced modulation methods for APF. These methods include the nearest level modulation (NLM) [12], phase-shifted PWM modulation (PSPWM) [13] and phase disposition PWM modulation (PDPWM) [14]. Nevertheless, NLM has limited applications because it cannot be used for multilevel converters with below 21 levels [15]. Meanwhile, PSPWM and PDPWM exhibit good performances in low-level converters, but require additional PI controllers to transfer the current reference to the output voltage, which may reduce the robustness of the system [16]. Moreover, these three methods have other disadvantages, including overshooting in current errors, sub-harmonic components in the current, limiting cycle oscillations, and non-optimum switching vector selection [17].

Hysteresis current tracking control has an inherent 


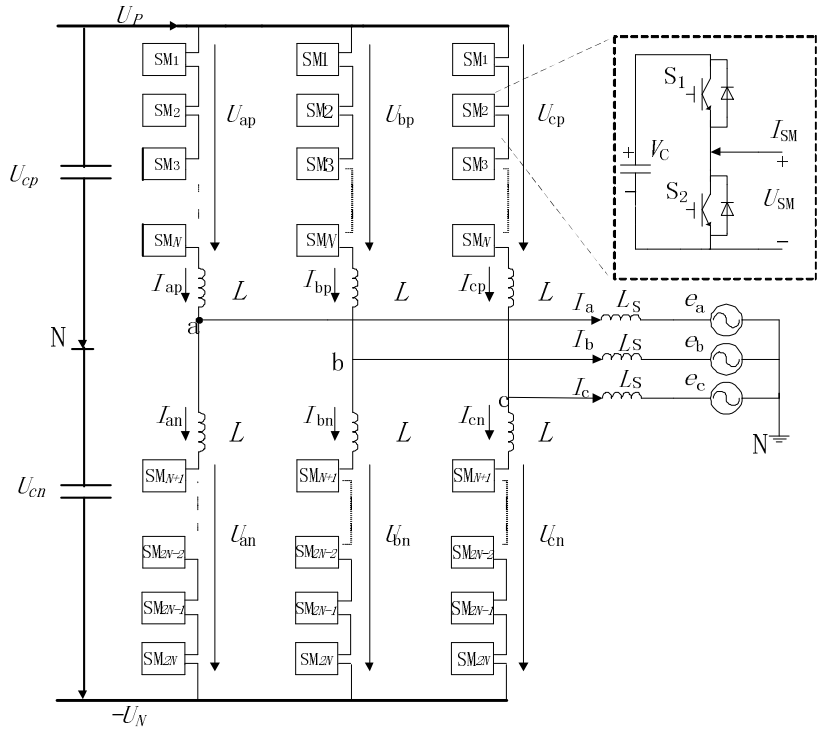

Fig. 1. Structure of a modular multi-level converter.

simplicity and rapid dynamic response, thus facilitating its wide use in many situations that require high harmonic current and current response rates, such as low-voltage APF [18] and reactive power compensation [19]. In this method, the output current of the converter, instead of the PI controllers of the inner loop in PSPWM and PDPWM, can be directly compared with the current reference. Hysteresis current tracking control is also a closed-loop control and has the advantages of high current response rate, fast real-time ability, and no need of carriers. Accordingly, applying this control in $\mathrm{MMC}$ is promising and can facilitate the implementations of MMC in middle and high voltage power systems.

However, due to the different topologies of MMC and common two-level inverters, applying the hysteresis current control in MMC may induce certain problems, including the unbalanced voltage of the capacitor, unbalanced voltage between the upper and lower arms, partition of the grid side voltage, and spectral characteristics of the current ripple.

This study intends to solve the above issues by proposing a quasi-fixed-frequency hysteresis current tracking control (QHCTC) strategy for MMC. In this novel strategy, the frequency of MMC output current is fixed, whereas the switching frequency of sub-module (SM) is quasi-fixed. In particular, the quasi-fixed-frequency is realized via virtual loop mapping (VLM), in which the SM switching frequency is similar to the output current frequency. This design can benefit the selection of switching devices and the assessment of system performances. The contributions of the proposed QHCTC are briefly described below.

First, by monitoring grid voltage and the deviation between the output and reference currents, the designed MMC can generate corresponding levels to force the output current to track the given current quickly and efficiently. In particular,
TABLE I

HALF-BRIDGe Sub-MOdule Working States

\begin{tabular}{c|c|c|c|c|c|c|c}
\hline \hline Mode & $\boldsymbol{S}_{\boldsymbol{k}}$ & $\boldsymbol{S}_{\boldsymbol{k} \boldsymbol{1}}$ & $\boldsymbol{S}_{\boldsymbol{k} \boldsymbol{2}}$ & $\boldsymbol{U}_{\boldsymbol{s} \boldsymbol{m}}$ & $\boldsymbol{I}_{\boldsymbol{s} \boldsymbol{m}}$ & $\begin{array}{c}\text { Stat } \\
\boldsymbol{e}\end{array}$ & $\begin{array}{c}\text { Capacit } \\
\boldsymbol{o r}\end{array}$ \\
\hline 1 & 1 & 1 & 0 & $V c$ & $>0$ & on & charge \\
\hline 2 & 1 & 1 & 0 & $V c$ & $<0$ & on & $\begin{array}{c}\text { discharg } \\
\text { e }\end{array}$ \\
\hline 3 & 0 & 0 & 1 & 0 & $>0$ & off & $\begin{array}{c}\text { no } \\
\text { change }\end{array}$ \\
\hline 4 & 0 & 0 & 1 & 0 & $<0$ & off & $\begin{array}{c}\text { no } \\
\text { change }\end{array}$ \\
\hline \hline
\end{tabular}

the non-average voltage partition principle is used for the current tracking to prevent the overlap of the output and grid voltages in every voltage region.

Second, varied hysteresis-band current tracking control is adopted by implementing fixed output current frequency on the basis of the above non-average voltage partition principle.

Third, the capacitor voltage of the upper/lower arm and the voltage between these arms are considered in the MMC design and are efficiently balanced with VLM and arm voltage balance (AVB), respectively. Accordingly, a balance control system and a quasi-frequency switching device driver are achieved.

Furthermore, the proposed method is validated with the simulations and experimental results of a five-level prototype of MMC.

\section{STRUCTURE AND OPERATION PRINCIPLES OF MMC}

\section{A. Basic Structure}

A basic three-phase four-leg MMC topology is shown in Fig. 1, in which the MMC is composed of three legs and six arms; each leg consists of one upper arm and one lower arm, and each arm contains a buffer inductor $L$ and $N$ series -connected SMs [21]. Also, phase voltages $U_{a}, U_{b}$, and $U_{c}$, are outputs from the intermediate connection point of the upper and lower arms, and their values depend on the difference between these arms.

In Fig. 1, SMs adopt the half-bridge sub-module (HBSM) design, which consists of a DC capacitor and two controllable power switches. HBSM is widely used in practice; its working status is shown in Table I where $S_{k 1}$ and $S_{k 2}$ are the driving signals of the upper and lower devices, respectively, whereas $V c$ is the average capacitor voltage of the $k^{\text {th }} \mathrm{SM}$.

Table I particularly illustrates that each SM has "1" and " 0 " statuses, and the corresponding output voltages $U_{s m}$ are $V c$ and 0 . In this case, "1" and " 0 " imply that SM is inserted and bypassed, respectively. When HBSM is inserted, the direction of $I_{s m}$ signifies the working status of the capacitor. If $I_{s m}>0$, the capacitor is charging, whereas if $I_{s m}<0$, the capacitor is discharging. 


\section{B. Operation Principles}

Since the operation principles of three legs (phase $j=a, b$, c) are comparable, this research selects and analyzes only one leg (phase $a$ ) of MMC. The Kirchhoff's voltage law demonstrates the below equations.

$$
\left\{\begin{array}{c}
U_{P}-U_{a N}=U_{a p}+L \frac{d I_{a p}}{d t} \\
U_{a N}-U_{N}=U_{a n}+L \frac{d I_{a n}}{d t} \\
U_{P}=-U_{N} \\
I_{a p}=I_{a n}+I_{a}
\end{array}\right.
$$

where $U_{P}$ and $U_{N}$ are DC bus voltages, $U_{a p}$ and $U_{a n}$ are the sum of the upper and lower arm capacitor voltages, respectively, $I_{a p}$ and $I_{a n}$ are the instantaneous current of the upper and lower arms, respectively, and $I_{a}$ is the output current of MMC.

By substituting (2) in (1), the below expressions are obtained.

$$
\begin{gathered}
U_{a N}=\frac{1}{2}\left(U_{a n}-U_{a p}\right)-\frac{L \cdot d I_{a}}{2 d t} \\
U_{a N}=L_{s} \frac{d I_{a}}{d t}+e_{a}(t)
\end{gathered}
$$

In traditional modulation methods, control systems provide the MMC modulation signals, which can be normalized and expressed as follows:

$$
U_{m j}=m \cos \left(\omega t+\varphi_{j}\right)
$$

where $m$ is the modulation index $(0 \leq m \leq 1)$, and $\varphi_{j}$ is the angular displacement of the fundamental frequency.

Without considering the circulating current suppression, the normalized reference voltage signals of the upper/lower arm should be demonstrated as follows:

$$
\begin{aligned}
& U_{m u}=\frac{1}{2}\left[1-m \cos \left(\omega t+\varphi_{j}\right)\right] \\
& U_{m d}=\frac{1}{2}\left[1+m \cos \left(\omega t+\varphi_{j}\right)\right]
\end{aligned}
$$

These signals, in which the effects of buffer inductor $L$ are ignored, have been widely used in MMC modulations such as NLM, PSPWM, and PDPWM. However, $L$ has to be considered in the hysteresis current tracking control. This condition will be analyzed in the next section.

\section{HYSTERESIS CURRENT TRACKING CONTROL STRATEGY}

\section{A. Hysteresis Current Tracking Control}

In general, grid-connected inverters have two control loops, namely, the outer voltage and inner current control loops, in which DC voltage regulation and current reference tracking are implemented, respectively. This research focuses on the latter and applies the hysteresis current tracking method to

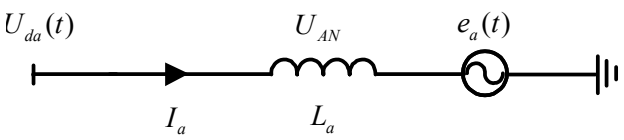

Fig. 2. Simplified single-phase MMC circuit.

MMC.

In particular, this study illustrates the principle of hysteresis current tracking control by simplifying the MMC model shown in Fig. 1 into the model depicted in Fig. 2, where $U_{d a}(t)$ is the equivalent output voltage, $L_{a}$ is the equivalent inductance, and $U_{A N}$ denotes the deviation between $U_{d a}(t)$ and grid voltage $e_{a}(t)$.

The simplified model above can be expressed by taking (3) into (4), as specified below.

$$
\begin{gathered}
\frac{1}{2}\left(U_{a n}-U_{a p}\right)-\frac{L}{2} \frac{d I_{a}}{d t}=L_{s} \frac{d I_{a}}{d t}+e_{a}(t) \\
U_{A N}=U_{d a}(t)-e_{a}(t) \\
L_{a}=L_{s}+\frac{L}{2} \\
U_{d a}(t)=\frac{1}{2}\left(U_{a n}-U_{a p}\right) \\
U_{A N}=L_{a} \frac{d I_{a}}{d t}
\end{gathered}
$$

Based on (8) to (12), Fig. 3 is generated to illustrate the principle of the hysteresis current tracking control, where $h$ is the hysteresis width, 0.5 and $-0.5 h$ denote the upper and lower thresholds, respectively, and $\Delta i_{j}$ is the deviation between output $I$ and reference currents $I_{j_{-}}$ref, as displayed in (13).

$$
\Delta i_{j}=I_{j_{-} r e f}-I_{j}, \quad j=a, b, c
$$

As signified in Fig. 3, when $\Delta i_{j}>0.5 h, I_{a}$ holds a downward trend with $U_{A N}<0$. Similarly, when $\Delta i_{j}<-0.5 h, I_{a}$ holds an upward trend with $U_{A N}>0$. Thus, $I_{a}$ is bounded within the hysteresis band, which is centered by the reference current. In practice, these operations can be implemented with the trigger signal of converter $D(t)$, which is defined below.

$$
\begin{cases}D(t)=0, & \Delta i_{j}>0.5 h \\ D(t)=1, & \Delta i_{j}<-0.5 h \\ D(t)=\text { unchange } & -0.5 h \leq \Delta i_{j} \leq 0.5 h\end{cases}
$$

Moreover, Fig. 3 indicates that $U_{A N}$ is critical to the performance of the hysteresis current tracking control because it determines the trend of $I_{a}$ and helps control the current within hysteresis bands. According to (9), generating the optimal $U_{A N}$ requires an effective design of the equivalent output voltage $U_{d a}(t)$ and grid voltage $e_{a}(t)$. In this research, the optimal $U_{A N}$ for $U_{d a}(t)$ is a staircase in (11) and is further expressed in (15). Meanwhile, the optimal $U_{A N}$ for $e_{\mathrm{a}}(t)$ is partitioned into $N+1$ regions because converter output voltage $U_{d a}$ has $N+1$ levels with $N+1$ level modulation from $L_{l}$ to 


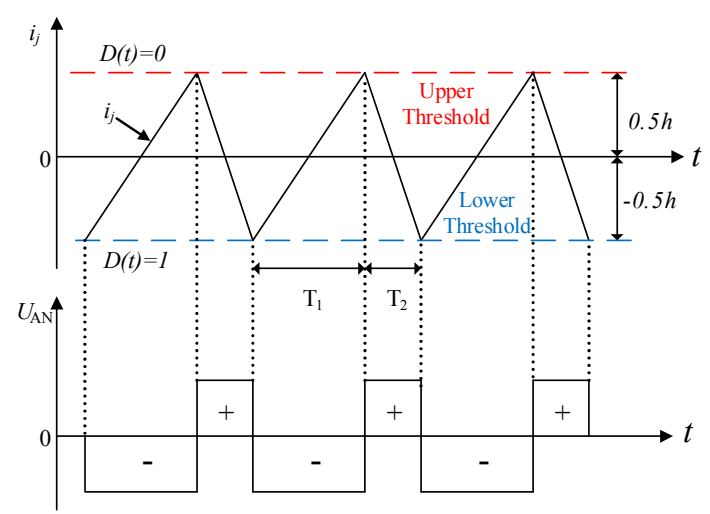

Fig. 3. Principle of hysteresis current tracking control.

$L_{N+1}$. Partitioned $e_{\mathrm{a}}(t)$ is expressed with the intermediate symbol $D(t)$ in (16).

$$
\begin{gathered}
\left\{\begin{array}{l}
U_{d a}(t)=\frac{1}{2}\left(U_{a n}-U_{a p}\right) \\
U_{a n}=\sum_{K=N+1}^{2 N} S_{K} \cdot V_{c} \\
U_{a p}=\sum_{K=1}^{N} S_{K} \cdot V_{c}
\end{array}\right. \\
\left\{\begin{array}{lr}
\frac{-U_{\mathrm{dc}}}{2} \leq \mathrm{e}_{a}(t)<\frac{V_{c}}{2}-\frac{U_{d c}}{2}, & V(t)=1 \\
\frac{V_{c}}{2}-\frac{U_{\mathrm{dc}}}{2} \leq \mathrm{e}_{a}(t)<\frac{3 V_{c}}{2}-\frac{U_{d c}}{2}, & \\
\cdots \cdots . . & \\
\left(M-\frac{3}{2}\right) V_{c}-\frac{U_{\mathrm{dc}}}{2} \leq \mathrm{e}_{a}(t)<\left(M-\frac{1}{2}\right) V_{c}-\frac{U_{d c}}{2}, & V(t)=\mathrm{M} \\
\cdots . . & V(t)=\mathrm{N}+1
\end{array}\right.
\end{gathered}
$$

Notably, the width of $1^{s t}$ and $N+1^{\text {th }}$ regions is $0.5 V_{C}$, while that of the others is $V_{C}$. This arrangement is designed to prevent misjudging the region of $e_{\mathrm{a}}(t)$, particularly at the vicinity of the converter output level.

An example of five-level converter is shown in Fig. 4 to demonstrate the relationship between the output voltage levels and grid voltage regions. In this figure, $e_{a \max }$ and $e_{\text {amin }}$ are the peak and trough values of $e_{\mathrm{a}}(t)$, respectively; $L_{0}$ to $L_{4}$ are the five output voltage levels of the MMC; and $V(t)$ represents the corresponding regions. The adjacent levels generating $U_{A N}$ in a specific region are grouped in common parentheses (e.g., working levels $\left(L_{2}, L_{4}\right)$ implies $V(t)=4$ ).

In addition, Fig. 4 presents the operation of the designed five-level MMC under the hysteresis current tracking control. When grid voltage $e_{\mathrm{a}}(t)$ exists in certain regions, the following cases emerge: 1) if $\Delta i_{j}$ exceeds the upper threshold $(D(t)=0)$, the MMC output voltage will be controlled to $U_{d a 2}(t)$ to reverse the current rising trend; 2) if $\Delta i_{j}$ decreases below the lower threshold $(D(t)=1)$, the MMC output voltage will be set to $U_{d a l}(t)$. Hence, the relationships of $D(t)$, $V(t)$, and $U_{d a}(t)$ can be summarized as denoted in Table II,

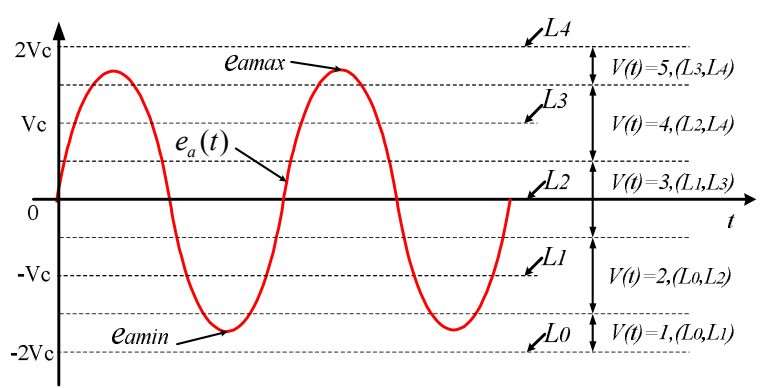

Fig. 4. Output voltage levels and grid voltage regions of five-level converter.

TABLE II

RELATIONSHIPS OF D(T), V(T), AND $\mathrm{U}_{\mathrm{DA}}(\mathrm{T})$

\begin{tabular}{c|c|l}
\hline \hline $\boldsymbol{V}(\boldsymbol{t})$ & $\boldsymbol{D}(\boldsymbol{t})$ & \multicolumn{1}{|c}{$\boldsymbol{U}_{d a}(\boldsymbol{t})$} \\
\hline \multirow{2}{*}{1} & 0 & $U_{d a 2}(t)=-U_{\mathrm{dc}} / 2$ \\
\cline { 2 - 3 } & 1 & $U_{d a 1}(t)=-U_{\mathrm{dc}} / 2+U_{\mathrm{dc}} / N$ \\
\hline \multirow{2}{*}{$\begin{array}{c}2,3, \ldots \\
\mathrm{N}\end{array}$} & 0 & $U_{d a 2}(t)=[V(t)-2] \cdot U_{\mathrm{dc}} / N-U_{\mathrm{dc}} / 2$ \\
\cline { 2 - 3 } & 1 & $U_{d a 1}(t)=V(t) \cdot U_{\mathrm{dc}} / N-U_{\mathrm{dc}} / 2$ \\
\hline \multirow{2}{*}{$\mathrm{N}+1$} & 0 & $U_{d a 2}(t)=U_{\mathrm{dc}} / 2-U_{\mathrm{dc}} / N$ \\
\cline { 2 - 3 } & 1 & $U_{d a 1}(t)=U_{\mathrm{dc}} / 2$ \\
\hline \hline
\end{tabular}

where $U_{d a 1}(t)$ and $U_{d a 2}(t)\left(U_{d a I}(t)>U_{d a 2}(t)\right)$ in each voltage partition represent the MMC output levels during the rise $T_{1}$ and fall times $T_{2}$, respectively.

Table II suggests that the number of the inserted lower arm SMs of the MMC is equal to $N(t)[21]$.

$$
\begin{cases}N(t)=0, & V(t)=1, D(t)=0 \\ N(t)=1, & V(t)=1, D(t)=1 \\ N(t)=V(t)+2 D(t)-2, & V(t) \neq 1, N+1 \\ N(t)=N-1, & V(t)=N+1, D(t)=0 \\ N(t)=N, & V(t)=N+1, D(t)=1\end{cases}
$$

\section{B. Performance Analysis}

The proposed method is further analyzed by evaluating the hysteresis control in a short-term period $T$, which consists of $T_{I}$ and $T_{2}$. By assuming that the switching frequency is sufficiently high and that the influence of fundamental frequency is ignored, the switching rise and fall times can be expressed as follows:

$$
\begin{gathered}
\left\{\begin{array}{l}
T_{1}=\frac{h \cdot L_{a}}{U_{A N}}=\frac{h \cdot L_{a}}{U_{d a 1}(t)-e_{a}(t)} \\
T_{2}=\frac{-h \cdot L_{a}}{U_{A N}}=\frac{h \cdot L_{a}}{e_{a}(t)-U_{d a 2}(t)}
\end{array}\right. \\
T=T_{1}+T_{2}=\frac{\left(h \cdot L_{a}\right)\left[U_{d a 1}(t)-U_{d a 2}(t)\right]}{\left[U_{d a 1}(t)-e_{a}(t)\right]\left[e_{a}(t)-U_{d a 2}(t)\right]}
\end{gathered}
$$

Accordingly, the output current frequency is

$$
f=\frac{1}{T}=\frac{\left[U_{d a 1}(t)-e_{a}(t)\right]\left[e_{a}(t)-U_{d a 2}(t)\right]}{\left(h \cdot L_{a}\right)\left[U_{d a 1}(t)-U_{d a 2}(t)\right]}
$$




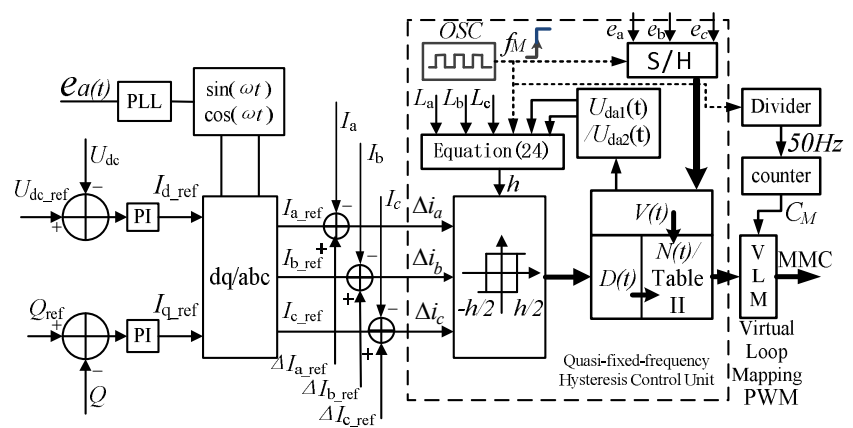

Fig. 5. Scheme of the quasi-fixed-frequency hysteresis current tracking control.

By combining the previous analysis in Table II, the below expressions are derived.

$$
\begin{gathered}
V(t)=1, \quad \text { with } \\
\left\{\begin{array}{l}
U_{d a 1}(t)-U_{d a 2}(t)=V_{c}=U_{d c} / N \\
f=\frac{\left[U_{d a 1}(t)-e_{a}(t)\right]\left[e_{a}(t)-U_{d a 1}(t)+V_{c}\right]}{\left(h \cdot L_{a}\right) V_{c}} \\
0.5 V_{c}<U_{d a 1}(t)-e_{a}(t) \leq V_{c}-0.5 U_{d c}-e_{a \min }
\end{array}\right. \\
V(t)=2,3, \ldots, N \quad \text { with } \\
\left\{\begin{array}{l}
U_{d a 1}(t)-U_{d a 2}(t)=2 V_{c}=2 U_{d c} / N \\
f=\frac{\left[U_{d a 1}(t)-e_{a}(t)\right]\left[e_{a}(t)-U_{d a 1}(t)+2 V_{c}\right]}{\left(h \cdot L_{a}\right) 2 V_{c}} \\
0.5 V_{c}<U_{d a 1}(t)-e_{a}(t) \leq 1.5 V_{c}
\end{array}\right. \\
V(t)=N+1, \quad w i t h \\
\left\{\begin{array}{l}
U_{d a 1}(t)-U_{d a 2}(t)=V_{c}=U_{d c} / N \\
f=\frac{\left[U_{d a 1}(t)-e_{a}(t)\right]\left[e_{a}(t)-U_{d a 1}(t)+V_{c}\right]}{\left(h \cdot L_{a}\right) V_{c}} \\
0.5 U_{d c}-e_{a \max } \leq U_{d a 1}(t)-e_{a}(t) \leq 0.5 V_{c}
\end{array}\right.
\end{gathered}
$$

Equations (21) to (23) indicate that the switching frequency of PWM signals for hysteresis control shows great uncertainty with a constant hysteresis width $h$. This issue induces difficulties in selecting switching devices and assessing system performance.

\section{Quasi-Fixed-Frequency Hysteresis Current Tracking Control}

The above challenges are tackled in this section by presenting the proposed QHCTC, which aims to obtain a fixed output current ripple frequency by controlling hysteresis width $h$ in real-time. A brief scheme of QHCTC is illustrated in Fig. 5, where $\mathrm{S} / \mathrm{H}$ represents the sample and hold function.

As depicted in Fig. 5, the MMC control system has two control loops, which are the outer DC voltage and the inner current control loops. The outer loop provides current references for the inner loop to utilize QHCTC, which adjusts the closed-loop current and transforms the current reference
TABLE III

True Value Table Of THE Lower ARM VSMs

\begin{tabular}{ccccccccc}
\hline $\boldsymbol{V}(\boldsymbol{t})$ & $\mathbf{1}$ & $\mathbf{2}$ & $\mathbf{3}$ & $\mathbf{4}$ & $\mathbf{5}$ & $\mathbf{6}$ & $\mathbf{7}$ & $\mathbf{8}$ \\
\hline 1 & $/ D(t)$ & 1 & 1 & 1 & $D(t)$ & 0 & 0 & 0 \\
\hline 2 & $/ D(t)$ & $/ D(t)$ & 1 & 1 & $D(t)$ & $D(t)$ & 0 & 0 \\
\hline 3 & $/ D(t)$ & $/ D(t)$ & 0 & 1 & $D(t)$ & $D(t)$ & 1 & 0 \\
\hline 4 & $/ D(t)$ & $/ D(t)$ & 0 & 0 & $D(t)$ & $D(t)$ & 1 & 1 \\
\hline 5 & $/ D(t)$ & 0 & 0 & 0 & $D(t)$ & 1 & 1 & 1 \\
\hline
\end{tabular}

signals into corresponding PWM switching signals. These tasks can be performed with the following procedures: 1) calculate the deviation currents $\Delta i_{a}, \Delta i_{b}$, and $\Delta i_{c}$ with (13); 2) generate $D(t)$ by comparing the deviation currents and hysteresis width $h$ in (24); 3) obtain $N(t)$ with (17). $D(t), V(t)$, and $N(t)$ are all available; hence, the proposed QHCTC can be implemented. From (20), hysteresis width $h$ can be acquired as follows:

$$
h=\frac{\left[U_{d a 1}(t)-e_{a}(t)\right]\left[e_{a}(t)-U_{d a 2}(t)\right]}{\left(f_{M} \cdot L_{a}\right)\left[U_{d a 1}(t)-U_{d a 2}(t)\right]}
$$

where $f_{M}$ is a fixed-frequency square wave similar to the system sample frequency.

In this study, the control process is simplified by expressing the calculation of (17) with the true value table. Consequently, the system efficiency is expected to be improved. In this case, the Virtual Sub-Module (VSM) [14, $20]$ is implemented, in which the driving signals are directly mapped from VSMs to RSMs. An $N=4$ example is illustrated, where the PWM signals of VSMs are arranged according to (17) and are listed in Table III. The VSMs of lower/upper arms are numbered from $l$ ' to $2 N$ '; 0 and 1 stand for "OFF" and "ON" status, respectively. The hysteresis result $D(t)$ is assigned to VSM 5' and VSM 6' when V(t) is equal to 2 to 4 , and $/ D(t)$ denotes the negated $D(t)$.

\section{BALANCE CONTROL SCHEME}

In this section, the issue of balancing arm capacitor voltages, which has not been covered in the above analysis, is solved by balancing the capacitor voltage of the upper/lower arm and the voltage between the upper and lower arms with VLM and AVB, respectively.

\section{A. Capacitor Voltage Balance Control}

VLM is applied to balance the arm capacitor voltage in each arm. VLM has been successfully used with PDPWM to balance the capacitor voltages of SMs [20]. The principle of VLM is to use a count-up counter $C_{M}$ for mapping VSMs to RSMs. The working frequency of $C_{M}$ can be set as required (in this paper, it is set as $50 \mathrm{~Hz}$ ), and its counting range is from 0 to $N-1$. The entire mapping process has been technically addressed by [14] and [20]. With this method, the capacitor voltage can be efficiently balanced in case of system symmetry (Selective Virtual Loop Mapping can also 


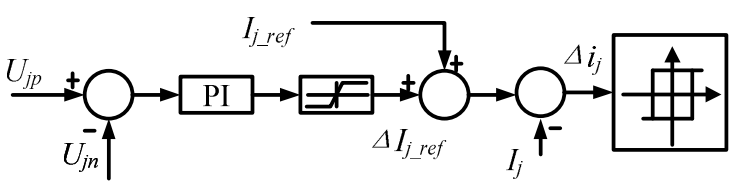

Fig. 6. Arm voltage balance control.

be used to acquire the capacitor voltage dynamic balance even if MMC loses its symmetry, but this issue is not the focus of this research).

After implementing VLM, the PWM signals will periodically combine the components of the fundamental and high order frequencies (e.g., fixed frequency $f_{M}$ ). This instance is the reason why the proposed method is named as "quasi-fixed-frequency hysteresis current tracking control."

\section{B. Arm Voltage Balance Control}

Apart from the unbalance voltage issue of a single arm, the unbalance voltages over the upper and lower arms should also be considered in MMC design. The unbalance voltages over the arms are mainly derived from the difference of energy losses among the arms and the zero drift error of MMC output current. Traditionally, the voltages of the arms are balanced by adding compensation into modulation signals [7]. This method, however, has the following disadvantages: 1) it is complex; 2) it requires PI controllers, which may reduce the robustness of the system; 3) its output waveform can be easily distorted; 4) it cannot be applied in hysteresis control. Therefore, this study implements the AVB [23]-[25] control method to address the abovementioned drawbacks. The block diagram of AVB is displayed in Fig. 6, where $U_{j p}$ and $U_{j n}$ are the sum of the upper and lower arm capacitor voltages $(j=a, b, c)$, respectively, and they can be replaced by capacitor voltages $U_{c p}$ and $U_{c n}$ in the single phase MMC.

In Fig. 6, the arm voltage balance correction signal $\Delta I_{j_{r} r e f}$ is generated by a PI controller, the input of which is the difference between $U_{j p}$ and $U_{j n}$, and is added to the current reference $I_{j \_}$ref, that can acquire the balance between $U_{j p}$ and $U_{j n .}$.

\section{SimUlations}

The proposed method is validated by implementing a simulation on the single-phase five-level MMC model. The schematic figure of the tested prototype is shown in Fig. 7 [20]. As depicted in Fig. 7, the model has four SMs in one arm, and the ground point is directly connected to the midpoint of two DC sources. In this case, $e_{a}(t)$ can be the grid voltage or the voltage across the load. The related system parameters are those specified in Table IV, in which the SM capacitor voltage is set as an average value and the influence of voltage ripple is neglected.

Fig. 8 shows the relationships of grid voltage $e_{a}(t)$, corresponding $V(t)$, and hysteresis width $h$ during $0.03 \mathrm{~s}$ to

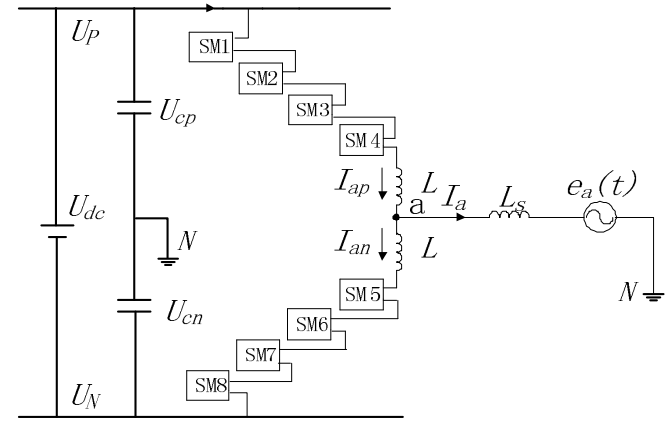

Fig. 7. Prototype of a single phase MMC.

TABLE IV

SimULATION PARAMETERS

\begin{tabular}{c|c|c|c}
\hline Parameters & Values & Parameters & Values \\
\hline DC link voltage $U_{d c}$ & $800 \mathrm{~V}$ & Arm inductor $L$ & $2 \mathrm{mH}$ \\
\hline $\begin{array}{c}\text { No. of SMs in each } \\
\text { arm }\end{array}$ & 4 & $\begin{array}{c}\text { Equivalent resistance of } \\
\text { arm } R\end{array}$ & $0.5 \Omega$ \\
\hline $\begin{array}{c}\text { SM capacitor voltage } \\
V c\end{array}$ & $200 \mathrm{~V}$ & Output current amplitude & $20 \mathrm{~A}$ \\
\hline SM capacitor $C$ & $2200 \mathrm{uF}$ & Counter frequency & $50 \mathrm{~Hz}$ \\
\hline $\begin{array}{c}\text { DC bus capacitor } C_{u p p}, \\
C_{u n}\end{array}$ & $4700 \mathrm{uF}$ & Sample frequency $f_{M}$ & $5 \mathrm{kHz}$ \\
\hline Output inductance $L_{S}$ & $6 \mathrm{mH}$ & Grid voltage amplitude & $311 \mathrm{~V}$ \\
\hline
\end{tabular}
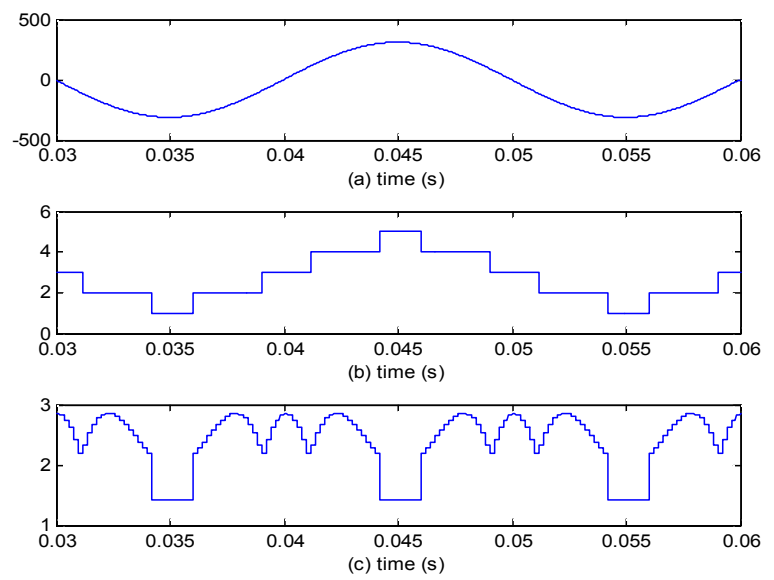

Fig. 8. Relationships of $e_{a}(t), V(t)$, and $h$.
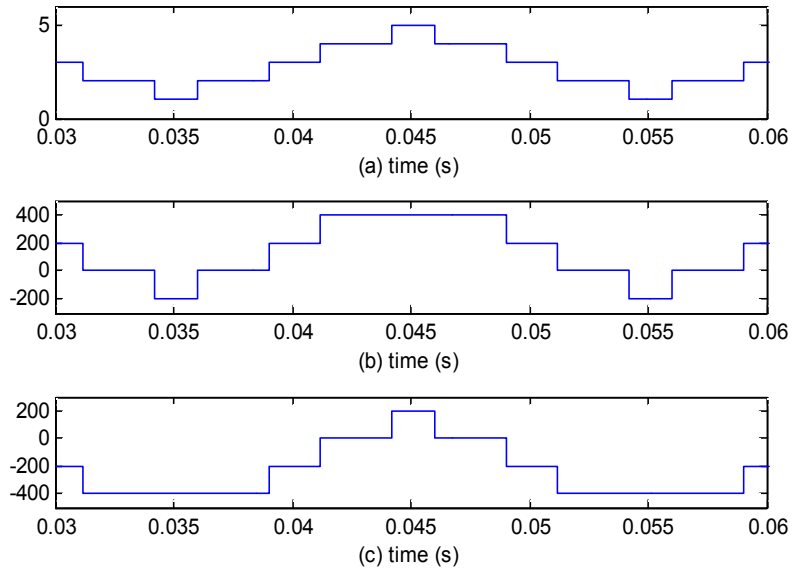

Fig. 9. $V(t)$ and the corresponding MMC output levels $U_{d a l}(t)$ and $U_{d a 2}(t)$. 

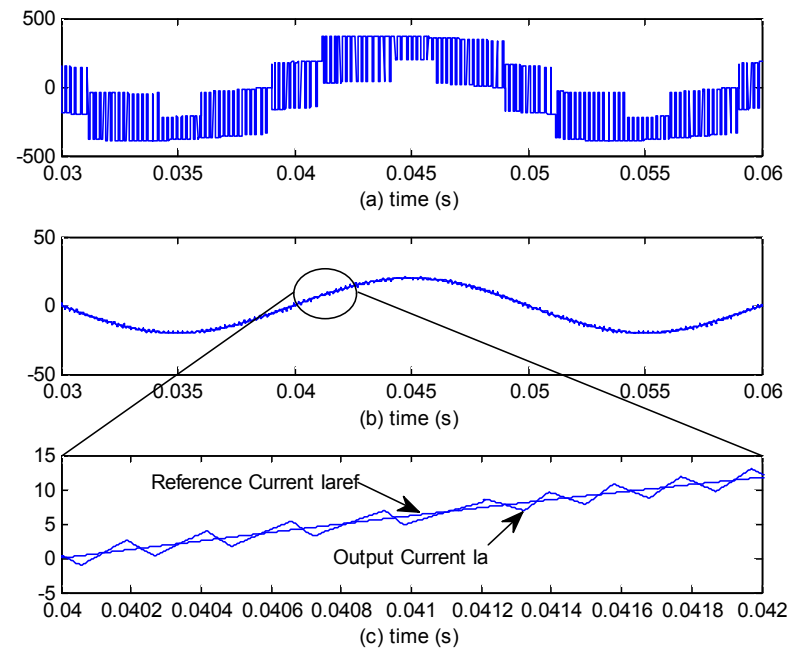

Fig. 10. Consequence of QHCTC: (a) $U a(t)$, (b) $I_{\text {aref }}$, and $I_{a}$, (c) and zoomed results between 0.04 and $0.042 \mathrm{~s}$.
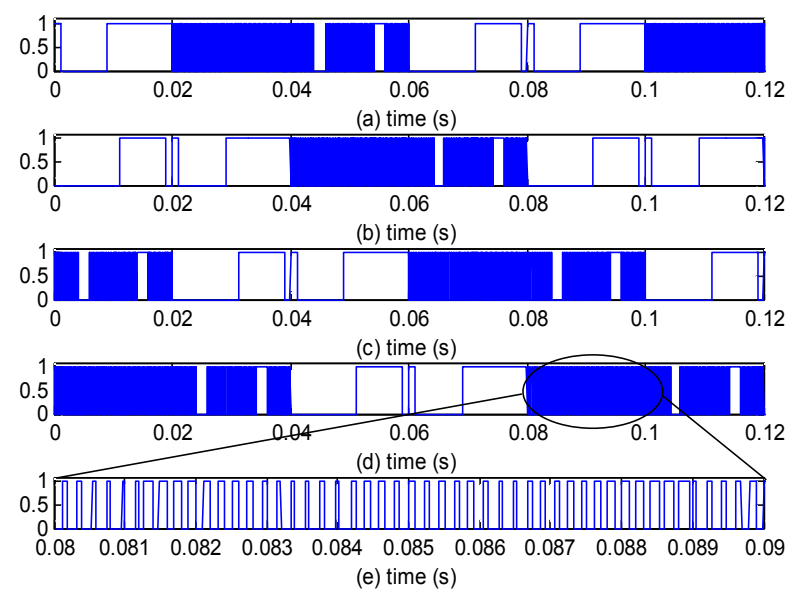

Fig. 11. PWM signals of SMs 1 to 4 between 0 and 0.12 s. (a) PWM1; (b) PWM2; (c) PWM3; (d) PWM4; (e) Zoomed PWM4 between 0.08 and $0.09 \mathrm{~s}$.

$0.06 \mathrm{~s}$. Hysteresis width $h$ is variable; thus, five regions $(-400$, $-300),(-300,-100),(-100,100),(100,300),(300,400)$ evidently exist, as indicated by $V(t)=1$ to $V(t)=5$, respectively.

Fig. 9 indicates the relationships among $V(t), U_{d a l}(t)$, and $U_{d a 2}(t)$. In particular, this figure demonstrates that $U_{d a 1}(t)>U_{d a 2}(t)$ holds in general, but the difference between $U_{d a 1}(t)$ and $U_{d a 2}(t)$ varies. Moreover, if $V(t)$ is equal to 1 or 5 , the voltage difference is $V_{c}$; otherwise, the voltage difference is $2 V_{c}$.

Fig. 10 presents the consequences of the proposed QHCTC. In particular, the MMC output voltage with reference current $I_{a_{-} \text {ref }}$ and MMC output current $I_{a}$ are shown in Figs. 10(a) and (b), respectively. Meanwhile, Fig. 10(c) is the zoomed results of Fig. 10(b) from $0.04 \mathrm{~s}$ to $0.042 \mathrm{~s}$, indicating that the actual current can efficiently follow the reference current with a fixed-frequency.

Figs. 11(a) to (d) exhibit the PWM signals of RSMs 1 to 4

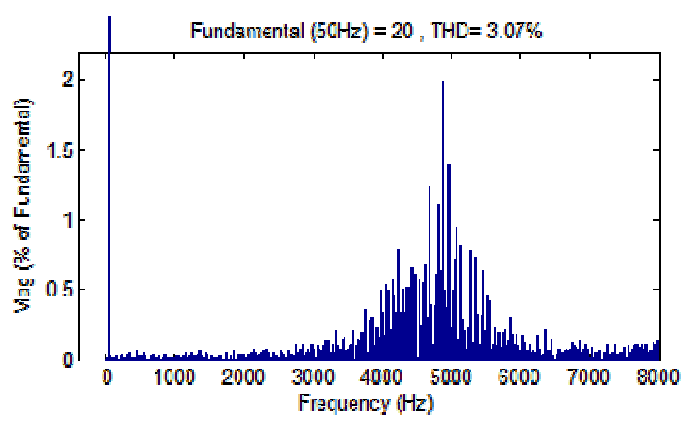

Fig. 12. Spectrum of output current $I_{a \text {. }}$

in the upper arm. These figures particularly illustrate that the frequency components in each PWM signal have two kinds, namely, the low-frequency and fixed high-frequency components. The low-frequency component depends on the PWM signals continuity, as specified in Table III. For example, when $V(t)$ is equal to 1,2 , and 3 , the PWM signals of VSMs 4' are all working in " 1 " state, whereas the PWM signals of VSMs 4' are working in " 0 " statewhen $V(t)$ is equivalent to 4 and 5 . Meanwhile, the high frequency component of PWM 4 is zoomed in Fig. 11(e), and its frequency is about $5 \mathrm{kHz}$, which is equal to $f_{M}$. A $0.02 \mathrm{~s}$ delay exists among these PWM signals due to the $50 \mathrm{~Hz}$ counter frequency of VLM.

Fig. 12 is the spectrum of $I_{a}$. In this case, THD is about $3.07 \%$, and the main frequency component is concentrated at nearly $5 \mathrm{kHz}$.

\section{EXPERIMENTS}

The feasibility of the proposed method is further verified by conducting single-phase experiments under low voltage on an MMC test bench. This time, grid voltage source $e_{a}(t)$ is replaced with a $15 \Omega$ resistor. The experimental parameters are listed in Table $\mathrm{V}$.

Fig. 13(a) shows the output voltage and current of the MMC in the case of five-level modulation, and Fig. 13(b) depicts the corresponding zoomed region of Fig. 13(a).

Fig. 14 reveals the output waveforms between PDPWM (VLM is used) and QHCTC. In particular, this figure evidently demonstrates that the latter has a smoother current waveform, particularly in the vicinity of the peak output current.

Fig. 15(a) describes the output voltage and corresponding PWM signal of one SM, and Fig. 15(b) is the corresponding zoomed region.

Meanwhile, Fig. 16 shows the MMC output voltage and current waveforms when the reference current amplitude and phase transitions simultaneously occur. The reference current amplitude changes from $6.2 \mathrm{~A}$ to $4 \mathrm{~A}$, accompanied by a $90^{\circ}$ phase shift. The result of the test indicates that the actual output current can rapidly track the reference current and demonstrates the high dynamic response capability of the proposed method. 
TABLE V

EXPERIMENTAL PARAMETERS

\begin{tabular}{c|c|c|c}
\hline \hline Parameters & Values & Parameters & Values \\
\hline DC link voltage $U_{d c}$ & $200 \mathrm{~V}$ & Arm inductor $L$ & $1.2 \mathrm{mH}$ \\
\hline $\begin{array}{c}\text { No. of SMs in each } \\
\text { arm }\end{array}$ & 4 & Load resistor & $15 \Omega$ \\
\hline Capacitor voltage $V c$ & $50 \mathrm{~V}$ & Sample frequency $f_{M}$ & $5 \mathrm{kHz}$ \\
\hline SM capacitor $C$ & $2200 \mathrm{uF}$ & $\begin{array}{c}\text { Output current } \\
\text { amplitude }\end{array}$ & $6.2 \mathrm{~A}$ \\
\hline $\begin{array}{c}\text { Output inductance } \\
L_{S}\end{array}$ & $4 \mathrm{mH}$ & Counter frequency & $50 \mathrm{~Hz}$ \\
\hline \hline
\end{tabular}

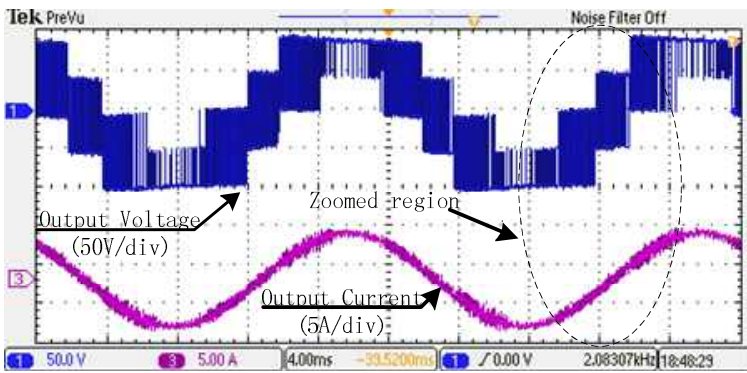

(a)

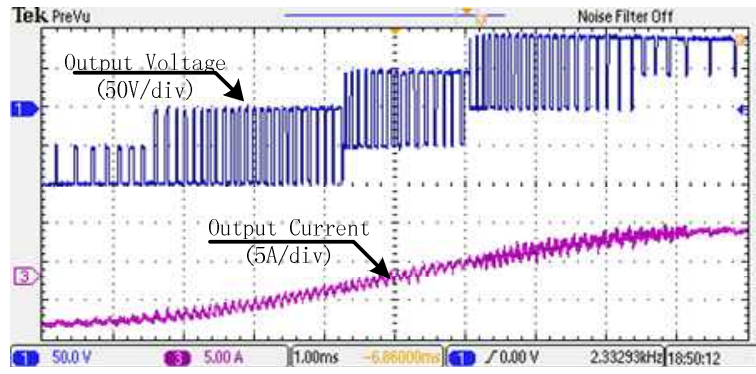

(b)

Fig. 13. (a) Output voltage and current of the experimental system. (b) Zoomed voltage and current waveforms of the experimental system

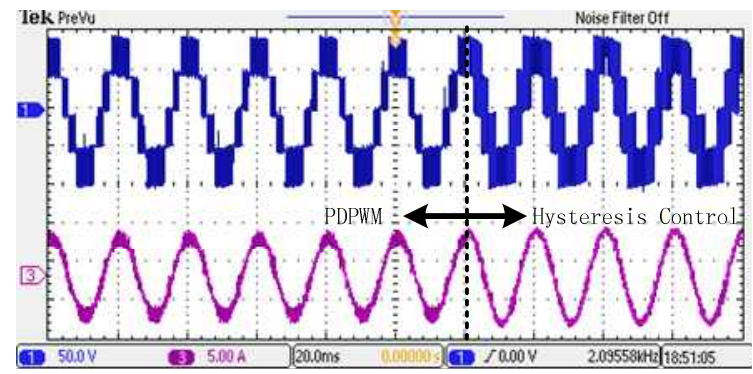

Fig. 14. Output voltage and current of the experimental system.

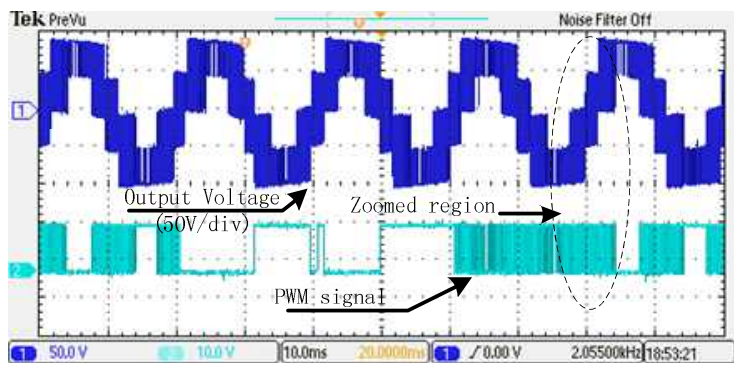

(a)

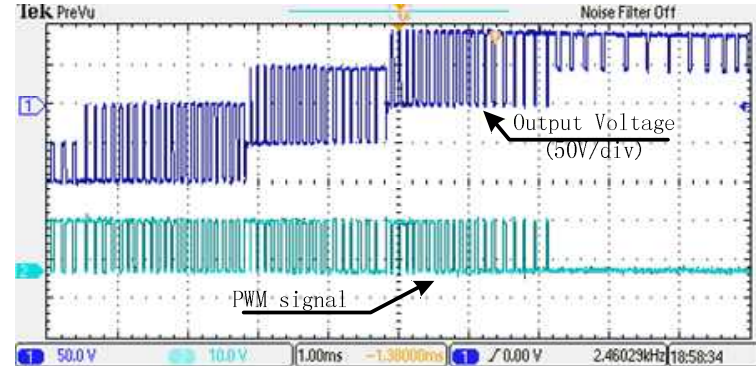

(b)

Fig. 15. (a) Output voltage and PWM signal. (b) Zoomed voltage and PWM signal waveforms.

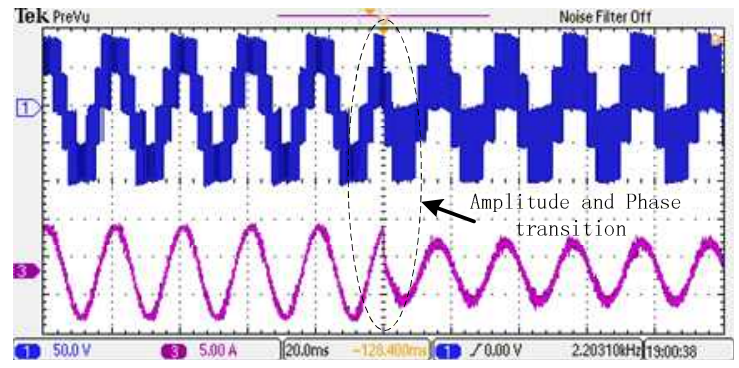

Fig. 16. Output voltage and current of the experimental system in the condition of the reference current's amplitude and phase transition

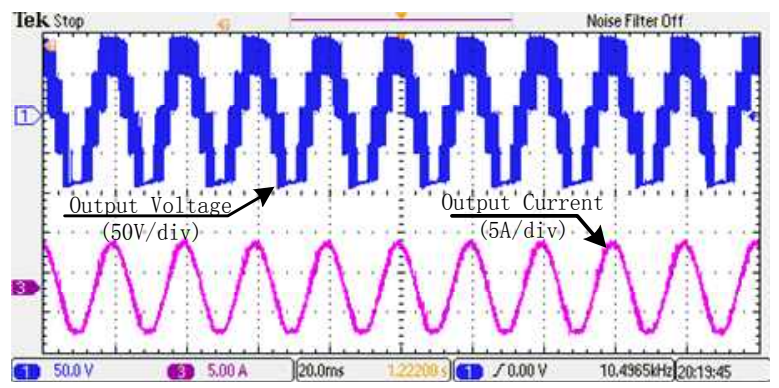

(a)

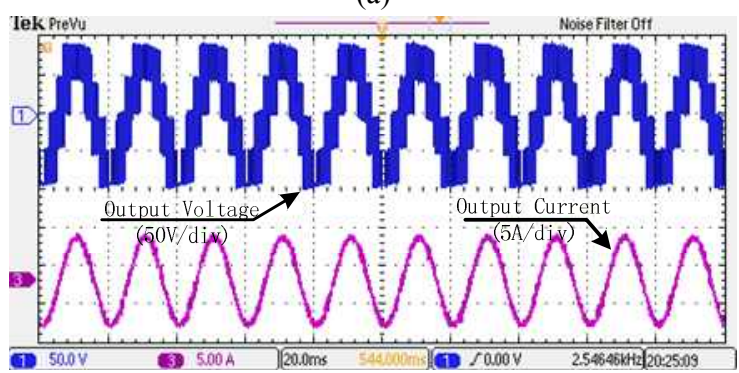

(b)

Fig. 17. (a) Output voltage and current when arm voltage loses balance. (b) Output voltage and current when arm voltage control is adopted.

In this experiment, the arm voltage balance control is also tested with the existence of some zero drift in the output current sensor. Fig. 17(a) illustrates the output voltage and current when the lower arm voltage is greater than the upper arm voltage. The output voltage and current distortion 
evidently occur in 17(a). Meanwhile, Fig. 17(b) presents the variation processes of the output voltage and current when the arm voltage control is applied. The results of the test show that the unbalance voltage of arms is effectively corrected.

\section{CONCLUSIONS}

This study proposes a new QHCTC strategy for MMCs based on the non-average voltage partition principle. The inserted number of the MMC lower arm SMs $N(t)$ is presented by derivation. By implementing both VLM and AVB, the capacitors' voltages of the upper/lower arms are efficiently balanced.

The proposed method is designed for any number and levels of SMs, and its validity is verified with the experimental results on a single-phase five-level MMC prototype.

\section{REFERENCES}

[1] S. P. Teeuwsen, "Modeling the Trans Bay Cable Project as voltage-sourced converter with modular multilevel converter design." in IEEE PES General Meeting, 2011.

[2] E. Solas, G. Abad, J. Barrena, S. Aurtenetxea, A. Carcar, and L. Zajac, "Modular multilevel converter with different submodule concepts - Part I: capacitor voltage balancing method," IEEE Trans. Ind. Electron., Vol. 60, No. 10, pp. 4525-4535, Oct. 2013.

[3] E. Solas, G. Abad, J. Barrena, S. Aurtenetxea, A. Carcar, and L. Zajac, "Modular multilevel converter with different subomodule concepts - Part II: experimental validation and comparison for HVDC application." IEEE Trans. Ind. Electron., Vol. 60, No. 10, pp. 4536-4545, Oct. 2013.

[4] G. Bergna, E. Berne, P. Egrot, P. Lefranc, A. Arzandé, J. Vannier, and M. Molinas, "An energy-based controller for HVDC modular multilevel converter in decoupled double synchronous reference frame for voltage oOscillation reduction," IEEE Trans. Ind. Electron., Vol. 60, No. 6, pp. 2360-2371, Jun. 2013.

[5] H. Saad, J. Peralta, S. Dennetière, J. Mahseredjian, J. Jatskevich, J. A. Martinez, A. Davoudi, M. Saeedifard, V. Sood, X. Wang, J. Cano, and Ali Mehrizi-Sani, "Dynamic averaged and sSimplified models for MMC-based HVDC transmission systems," IEEE Trans. Power Del., Vol. 28, No. 3, pp. 1723-1730, Jul. 2013.

[6] G. Bergna, E. Berne, P. Egrot, P. Lefranc, A. Amir, J. Vannier, and M. Molinas, "An energy-based controller for HVDC modular multilevel converter in decoupled double synchronous reference frame for voltage oscillations reduction," IEEE Trans. Ind. Electron., Vol. 60, No. 6, pp. 2360-2371, Jun. 2013.

[7] M. Hagiwara, K. Nishimura, and H. Akagi, "A mediumb -voltage motor drive with a modular multilevel PWM inverter," IEEE Trans. Power Electron, Vol. 25, No. 7, pp. 1786-1799, Jul. 2010

[8] H. P. Mohammadi, and M. T. Bina, "A transformerless medium-voltage STATCOM topology based on extended modular multilevel converters," IEEE Trans. Power Electron, Vol. 26, No. 5, pp. 1534-1545, May 2011.

[9] M. Hagiwara, R. Maeda, and H. Akagi. "Negative -sequence reactive-power control by a PWM STATCOM based on a modular multilevel cascade converter (MMCC -SDBC)," IEEE Trans. Ind. Appl., Vol. 48, No. 2, pp. 720 -729, Apr. 2012

[10] S. Rajasekar and R. Gupta, "Solar photovoltaic power conversion using modular multilevel converter," in Proc. Engineering and Systems (SCES 2012), 2012.

[11] F. T. Ghetti, A. A. Ferreira1, H. A. C. Braga, and P. G. Barbosa, "A study of shunt active power filter based on modular multilevel converter (MMC)," in Proc. 10th IEEE /IAS Industry Applications (INDUSCON 2012), 2012.

[12] N. Ahmed, A. Haider, L. Angquist, and H.-P. Nee, "M2C -based MTDC system for handling of power fluctuations from offshore wind farms," in Proc. IET Renewable Power Generation (RPG 2011), 2011

[13] S. Rohner, S. Bernet, M. Hiller, and R. Sommer, "Modulation, loses, and semiconductor requirements of modular multilevel converters," IEEE Trans. Ind. Electron., Vol. 57, No. 8, pp. 2633-2642, Aug. 2010.

[14] J. Mei, K. Shen, B. Xiao, and L. Tolbert, "A new selective loop bias mapping phase disposition PWM with dynamic voltage balance capability for modular multilevel converter," IEEE Trans. Ind. Electron., Vol. 61, No. 2, pp. 798-807, Feb. 2013.

[15] Q. Tu, Z. Xu, and W. Yao, "Selecting number of voltage levels for modular multilevel converter based HVDC," Power System Protection and Control, Vol. 38, No. 20, pp. 33-38, 2010. (in Chinese)

[16] T. Ghennam, E. M. Berkouk, and B. Francois, "A novel space-vector current control based on circular hysteresis areas of a three-phase neutral-point-clamped inverter," IEEE Trans. Ind. Electron., Vol. 57, No. 8, pp. 2669-2678, Jul. 2010.

[17] A. Dey, P. P. Rajeevan, R. Ramchand, K. Mathew, and K. Gopakumar, "A space-vector-based hysteresis current controller for a general n-level inverter-fed drive with nearly constant switching frequency control," IEEE Trans. Ind. Electron., Vol. 60, No. 5, pp. 1989-1998, May 2013.

[18] H. Vahedi, E. Pashajavid, and K. Al-Haddad, "Fixed-band fixed-frequency hysteresis current control used In APFs," in Proc. 38th Annual Conference on IEEE Industrial Electronics Society (IECON 2012), pp. 5944-5948, 2012.

[19] A. Shukla, A. Ghosh, A. Joshi, "Hysteresis current control operation of flying capacitor multilevel inverter and its application in shunt compensation of distribution systems," IEEE Trans. Power Del., Vol. 22, No. 1, pp. 396-405, Jan. 2007.

[20] J. Mei, B. Xiao, K. Shen, L. Tolbert, and J. Y. Zheng, "Modular multilevel inverter with new modulation method and its application to photovoltaic grid-connected generator," IEEE Trans. Power Electron, Vol. 11, pp, 5063-5073, May 2013.

[21] Y. Suresh, A. K. Panda, M. Suresh, "Real-time implementation of adaptive fuzzy hysteresis-band current control technique for shunt active power filter," IET Power Electron., Vol. 5, No. 7, pp. 1188-1195, May 2012.

[22] L. Zhang, M. J. Waite, and B. Chong, "Three-phase four-leg flying-capacitor multi-level inverter-based active power filter for unbalanced current operation," IET Power Electron., Vol. 6, No. 1, pp. 153-163, Jan. 2013.

[23] M. Hagiwara and H. Akagi, "Control and experiment of pulsewidth- modulated modular multilevel converters," IEEE Trans. Power Electron., Vol. 24, No. 7, pp. 1737746, Jul. 2009.

[24] H. Akagi, "Classification, terminology, and application of the modular multilevel cascade converter (MMCC)," IEEE 
Trans. Power Electron., Vol. 26, No. 11, pp. 3119-3130, Nov. 2011.

[25] K. Wang, Y. Li, Z. Zheng, and L. Xu, "Voltage balancing and fluctuation suppression method of floating capacitors in a new modular multilevel converter," IEEE Trans. Ind. Electron., Vol. 60, No. 5, pp. 1943-1954, May 2013.

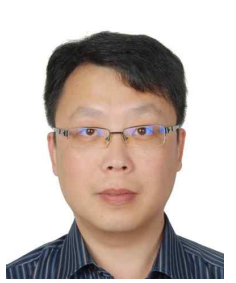

Jun Mei received his B.S. degree in radio engineering from the Chongqing University in 1994 and his M.S. and Ph.D. degrees in electrical engineering from the Southeast University, Nanjing, China, in 2001 and 2006, respectively. At present, he is an Associate Professor in the Department of Electrical Engineering, Southeast University. His interests include electric power converters for distributed energy sources, FACTS, and power quality control.

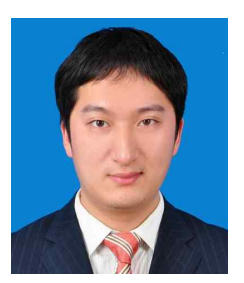

Yu Ji received his B.S.E.E and M.S.E.E. degrees from the Southeast University, Nanjing, China, in 2009 and 2012, respectively. $\mathrm{He}$ is currently pursuing his $\mathrm{Ph} . \mathrm{D}$. degree in electrical engineering at the same academe. His current research interests include the application of power electronics in power system, voltage source converter-based HVDC, controlling technique and developing the multilevel inverters of HVDC.

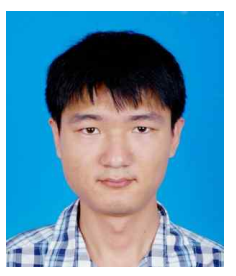

Xiaozhou Du received his B.S.E.E degree from Anhui University, Hefei, China, in 2012, and is currently pursuing M.S.E.E. in electrical engineering at Southeast University, Nanjing, China. His current research interests include the application of power electronics in power system and voltage source converter-based HVDC.

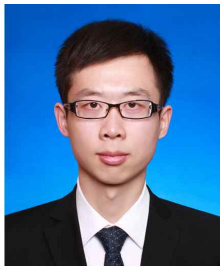

Tian Ma received his B.S.E.E degree from Southeast University, Nanjing, China, in 2013, and is currently pursuing M.S.E.E. in electrical engineering in Southeast University, Nanjing, China. His current research interests include the application of power electronics in power system and voltage source converter-based HVDC.

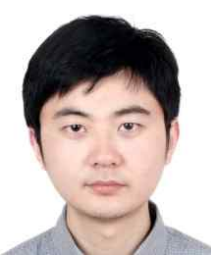

Can Huang received his B.S.E.E degree from Hohai University, Nanjing, China, in 2008, and M.S.E.E. degree from Southeast University, Nanjing, China, in 2011. He was with the State Grid Electric Power Research Institute (NARI Group Corporation), Nanjing, China, from 2011 to 2012. Currently, he is a $\mathrm{Ph} . \mathrm{D}$. candidate in electrical engineering at the University of Tennessee, Knoxville, TN, USA. His research interests include energy conversion and integration, power system operation and planning, and IT applications in power system measurement, protection. and communication.

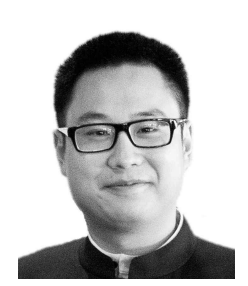

Qinran Hu received his B.S.E.E. degree from Southeast University, Nanjing, China, in 2010, and M.S.E.E. degree from the University of Tennessee, Knoxville, TN, USA, in 2013. He is currently pursuing his $\mathrm{Ph} . \mathrm{D}$. degree in electrical engineering at the University of Tennessee. His research interests include renewable energy integration, smart energy management system, and power system optimization. 